\title{
Realisation of Sensitive Functionality by Integration of Electromagnetic Resonators in Composite Materials
}

Toni D. Großmann ${ }^{1}$, Melinda Hartwig ${ }^{1)}$, Michael Heinrich ${ }^{2)}$, Ricardo Decker ${ }^{1)}$, Christina Symmank ${ }^{1)}$, Anja Schmidt ${ }^{1)}$, Steffen Kurth ${ }^{3)}$, Uwe Götze ${ }^{1}$, Reinhard R. Baumann ${ }^{1,3)}$, Lothar Kroll ${ }^{1,2)}$, Thomas Otto ${ }^{1,3)}$

1) Chemnitz University of Technology, toni-dirk.grossmann@zfm.tu-chemnitz.de, melinda.hartwig@mb.tu-chemnitz.de, ricardo.decker@mb.tu-chemnitz.de, christina.symmank@wirtschaft.tu-chemnitz.de, anja.schmidt@wirtschaft.tu-chemnitz.de, uwe.goetze@wirtschaft.tu-chemnitz.de, reinhard.baumann@mb.tu-chemnitz.de, lothar.kroll@mb.tu-chemnitz.de, thomas.otto@etit.tu-chemnitz.de, Chemnitz, Germany

2) Fraunhofer Institute for Machine Tools and Forming Technology IWU, michael.heinrich@iwu.fraunhofer.de, Chemnitz, Germany

3) Fraunhofer Institute for Electronic Nano Systems ENAS, steffen.kurth@enas.fraunhofer.de, reinhard.baumann@enas.fraunhofer.de, thomas.otto@enas.fraunhofer.de, Chemnitz, Germany

\section{Keywords}

Business model, Electromagnetic resonators, Smart structures, Stand-off detection, Structural health monitoring.

\begin{abstract}
Lightweight structures are gaining importance due to the relevance of saving energy in mobile applications. External stress caused by impacts, deformations or compression injures the composite materials mostly by invisible internal distortions and leads to the degradation of their properties. Thus, an early detection of material damage is significant in applications with a very high level of required reliability. Structural health monitoring (SHM) on demand using functionalised materials can be a solution [1, 2]. The integration of electromagnetic resonators in glass-fibre-reinforced plastics (GFRP) allows the fabrication of materials with passive sensor function used for SHM of composite materials. Conductive patterns with a specific geometry, dimension and alignment show an electromagnetic resonance that can be changed by the arrangement of the resonators or by the surrounded material. Printing technology is an efficient fabrication method regarding resources, time consumption and costs. The additive and selective deposition of conductive ink on flexible substrates shows a great potential to be processed roll-to-roll and subsequently integrated into lightweight structures [3]. The read-out takes place wirelessly by analysing the reflection response of the functionalised structure. The paper considers the modelling, numerical analysis, fabrication and evaluation of a smart structure and its sensor function. Furthermore, in order to create a basis for a successful market introduction and penetration of such innovative smart structures, a concept for an integrated life cycle-related engineering and business modelling [4] is outlined in this paper.
\end{abstract}

\section{Introduction and Motivation}

Remote and contactless monitoring of material and structure properties is a technical challenge with high application benefit. The early detection of elastic as well as inelastic deformations and the detection of cracks and delamination plays an important role, especially in the area of composite materials, to allow predictions about their structural integrity and reliability. By means of locally placed active sensors with integrated electronics and radio transmission capabilities, a permanent monitoring of lightweight structures is possible. In $[5,6]$ integrated piezoelectric actuators and sensors are shown. The ultrasonic waves produced by the piezoelectric actuators propagate on top of the surface of the monitored structure as guided waves. Piezoelectric sensors receive the modified guided waves caused by defects in the composite material. Passive sensor approaches provide another option. They do not need active 
electrical power for their operation and are less expensive in fabrication. A concept for a passive sensor approach is presented in [7]. They use a dipole antenna placed on a plastic substrate and operating in the $x$-band to detect material strain by varying the capacitive load at the feeding point of the antenna caused by the applied strain. Another approach shown in [8] and [9] uses surface acoustic wave (SAW) sensors. Applying a strain to the sensor chip that contains the SAW chip influences the resonance frequency. However, the sensor ranges of the named sensor approaches are locally restricted and the integrated sensors lead to local defects causing undesired stress concentrations in case of rising material load. Moreover, the permanent mechanical connection of the sensors is still a challenge.

The application and integration of electromagnetic resonators into the observing structure overcome the mentioned difficulties and enable the creation of an artificially designed material (metamaterial). This allows for spatial distribution of the sensory function within the material and eliminates the sensitivity limitations of locally integrated sensors. In [10] single split ring resonators (SSR) and double split ring resonators (DSSR) are used to investigate a metamaterial-based wireless strain sensor.

This paper investigates the application of metamaterials within lightweight structures made of GFRP to achieve a smart material with passive sensing capabilities used for SHM by the integration of electromagnetic resonator arrays into a lightweight structure. Printing technology is applied to form a periodic and regular array of electromagnetic resonators on a polymer substrate and it creates a compliant and regular arrangement of the resonators into the structures that are to be monitored.

This paper reports on the design, the numerical analysis and the realisation of the resonator arrays and shows the fabrication sequence as well as the integration of the arrays into a GFRP structure. Moreover, it reflects the results obtained from the experiments to evaluate the sensor approach. In contrast to conventional sensor technologies, i.e. SAW, strain gauge or fibre Bragg grating, the approach of using printed resonators allows for the reduction of the fabrication effort and the costs of the integration process. In order to fully deploy the potential of this innovative technology, technical research and engineering activities should be integrated with business modelling. Therefore, an appropriate concept is presented and the first step of the business modelling part is addressed.

\section{Approach, Design and Realisation}

Electromagnetic resonators (resonators) made of conductive material show a specific electromagnetic behaviour in the gigahertz range. The alteration of the geometry, dimension, alignment and arrangement allows for the modification of the electromagnetic behaviour, especially the resonance frequency of the resonators. In addition, materials near the resonator can influence the resonance behaviour. The option to change the arrangement of the resonators or to affect the material constitution around them allows the well-directed generation of a sensor function. Consequently, the integration of electromagnetic resonators within composite materials allows their functionalisation and thus the realisation of smart structures with passive sensor function.

\subsection{Approach}

The sensor approach uses two resonator arrays. Those will be integrate into a lightweight structure to influence the reflection and transmission behaviour. The application of resonators leads to the formation of resonance peaks and resonance dips that are visible in the reflection or transmission response. The occurrence of the resonance peaks and dips depend on the geometry, dimensions, alignment and arrangement of the resonators as well as on the material properties around them. This paper investigates the arrangement of two resonator arrays integrated into a lightweight structure in order to analyse their reflection behaviour in case of load. The applied material stress results in a structural change, causes a slight shift in the alignment of the resonators with each other and influences the distance between the two resonator arrays. This causes a frequency shift of the resonances and antiresonances.

The sensor approach was evaluated using CST Microwave Studio $\AA$ [Computer Simulation Technology AG]. The software enables the investigation of the electromagnetic behaviour of the resonator array in combination with the lightweight structure. The CST software considers an expansively expanded structure of the same shape (array) looking only at a single element of that array (unit cell). 


\subsection{Design}

In order to evaluate this concept, two resonator arrays composed of 400 single split ring resonators (SSR) are arranged on a flexible substrate (PET foil) of thickness $100 \mu \mathrm{m}$ and with a pitch of $4 \mathrm{~mm}$. The outer length of one SSR resonator is $1.8 \mathrm{~mm}$, the conductor width is $0.4 \mathrm{~mm}$, and the gap is $0.5 \mathrm{~mm}$ (Figure $1 \mathrm{a}, \mathrm{b}$ ). The conducting material is silver with a conductivity of $6.3 \times 10^{7} \mathrm{~S} / \mathrm{m}$ (typical value for Silver). Two resonators on substrate with equal shape are arranged in a parallel configuration face to face to form the unit cell of the array with an intermediate layer of GFRP of $0.9 \mathrm{~mm}$ thickness (Figure 1a and Figure 1c). Moreover, two further layers of GFRP material are considered by the model in order to simulate a structure which is more relevant for possible applications. A numerical analysis was performed to characterise the basic reflection and transmission behaviour and to determine the significant resonance peaks and dips of the structure.

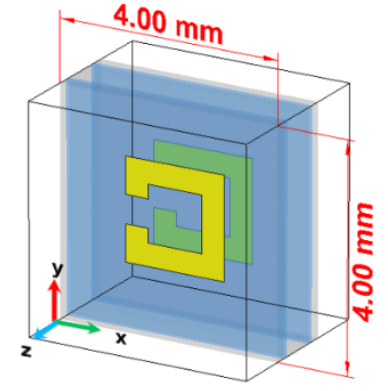

a)

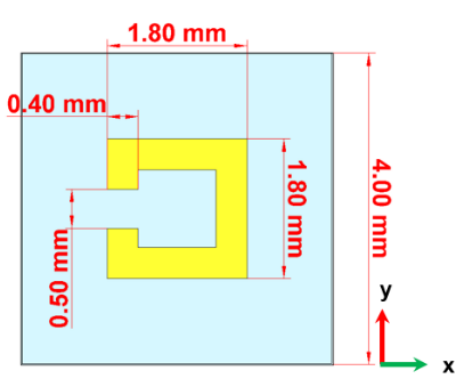

b)

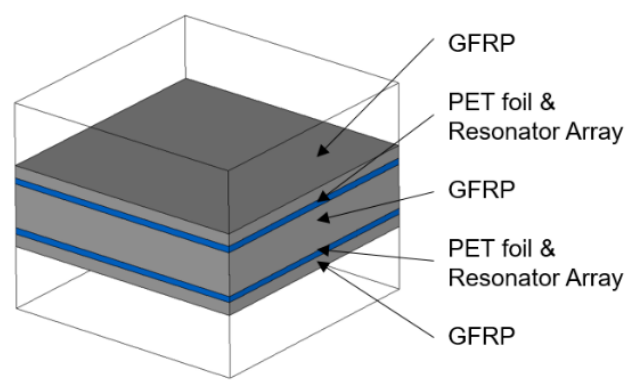

c)

Figure 1: Simulation model of $a, b)$ the considered unit cell with the SSR resonators and c) schematic view of the investigated layer stack

From the reflection behaviour of the above described layer stack, one can conclude the following statements:

The application of strain to the FGFRP in y-direction leads to an elongation as well as to a decrease in the thickness of the structure, caused by lateral contraction. With the elongation of the considered material stack, it is expected that the distance between the neighbouring resonators within one resonator array is increased in the direction of the acting force. To analyse this effect and the impact on the electromagnetic behaviour of the functionalised structure, a numerical simulation was performed, and its results are shown in Figure $2 a$.

In case of a thickness reduction due to lateral contraction, it is expected that the two resonator arrays will reduce their distance to each other. This issue was also analysed numerically and is shown in Figure $2 \mathrm{~b}$.

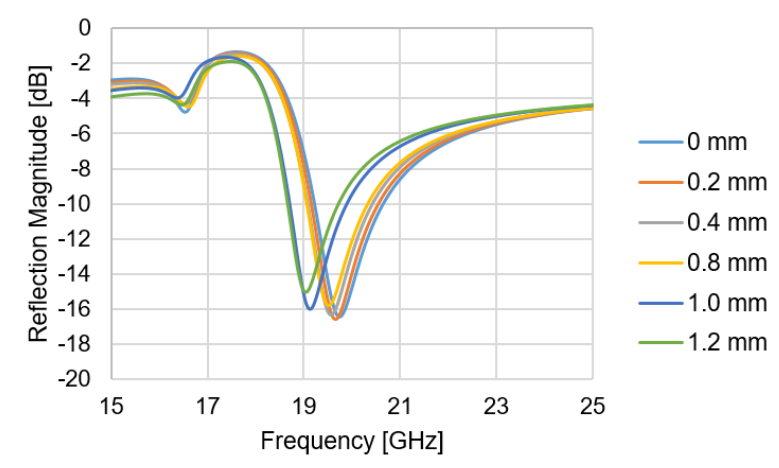

a)

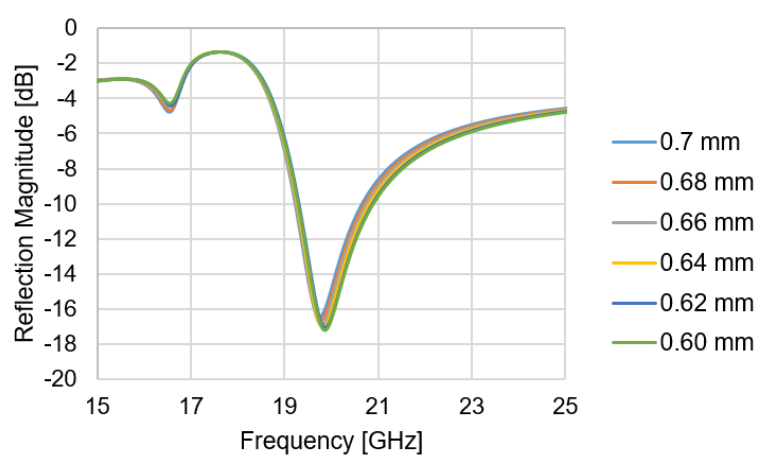

b)

Figure 2: Numerical analysis of the reflection response in case of a) stretching and b) in case of a decreasing distance between the two resonator arrays 
Figure $2 a$ shows the reflection magnitude of the investigated FGFRP structure in the frequency range from $15 \mathrm{GHz}$ to $25 \mathrm{GHz}$. The reflection response depicts two reflection dips in the vicinity of $17 \mathrm{GHz}$ and $20 \mathrm{GHz}$ that enclose a broadband reflection peak in the range between $16.5 \mathrm{GHz}$ and $18.5 \mathrm{GHz}(-3 \mathrm{~dB}$ upper and lower frequencies). The formation shows a stopband characteristic. Furthermore, the first reflection dip close to $17 \mathrm{GHz}$ appears weaker in magnitude than that close to $20 \mathrm{GHz}$. The analysis of the simulation results show that the reflection dip at $17 \mathrm{GHz}$ depends on the distance to the second SSR resonator array. If the distance between the two resonator arrays is further decreased, the magnitude of the first dip also decreases and finally disappears. On the other hand, if the distance is increased, the magnitude of the first dip increases and shifts to lower frequencies. The second reflection dip close to $20 \mathrm{GHz}$ can be attributed to the anti-resonance that is caused by destructive interference due to the interaction between the resonators and the external driven time varying electromagnetic field. The antiresonance shifts to higher frequencies (Figure $2 b$ ) when the distance between the two resonator arrays is decreased. This is the case when the material thickness becomes smaller.

The consideration of the reflection peak shows that here most of the incident power is reflected back from the structure. This is because of the first resonance order of the resonator array. Figure 3 shows the averaged electric and magnetic field distribution at $17.952 \mathrm{GHz}$ related to the first resonance order.

The first order resonance mode of the SSR resonator causes an excessive electric field in the gap region and a strong magnetic field in the middle section.
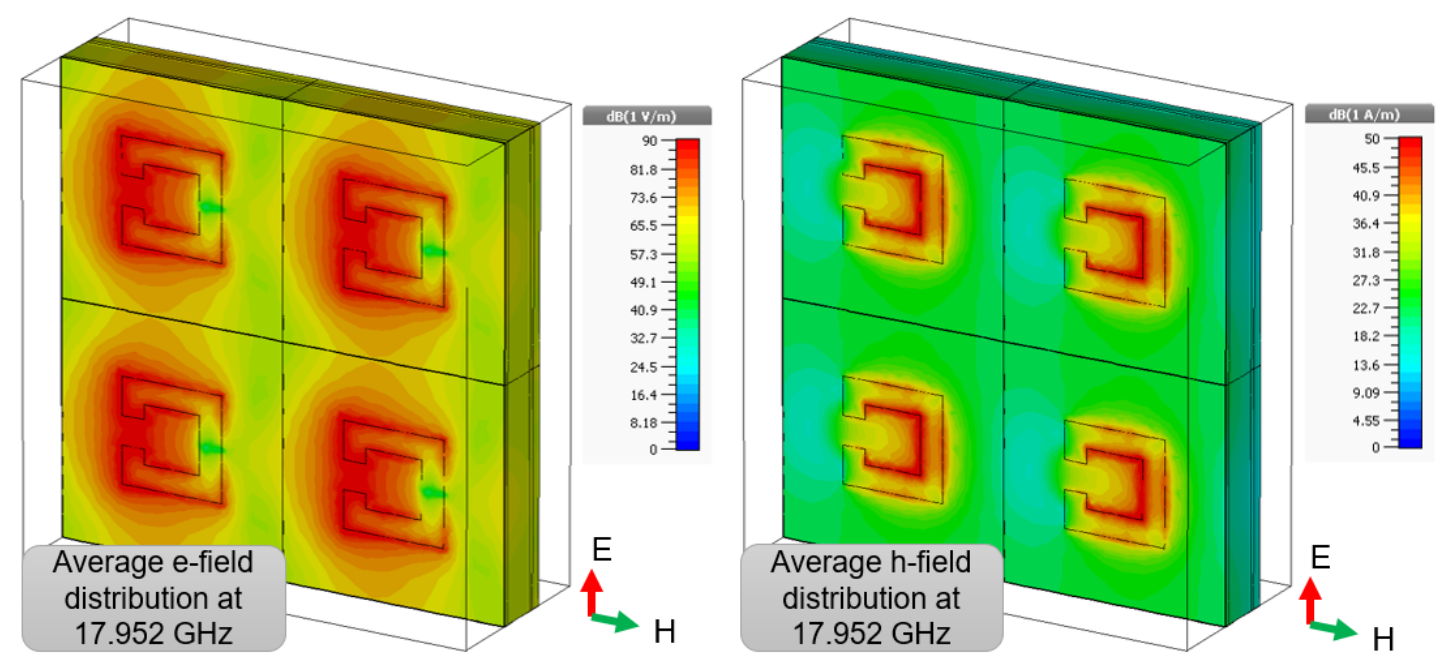

Figure 3: Plots of the average electric (left) and magnetic field (right) distribution at 17.952 GHz. The directions of the exciting $\mathrm{E}$ - and $\mathrm{H}$-field plots.

Furthermore, Figure 2a shows that the reflection peak and the reflection dip at $20 \mathrm{GHz}$ shift slightly to lower frequencies when the distance between the resonators within the resonator array increases from $0 \mathrm{~mm}$ to $1.2 \mathrm{~mm}$. In addition, a larger frequency shift occurs at the transition from $0.8 \mathrm{~mm}$ to $1.0 \mathrm{~mm}$.

\subsection{Realisation}

\subsubsection{Gravure Printing Technology}

Roll-to-roll gravure printing technology was used for the realisation of the resonators. This is an indirect process where the pattern is inversely engraved into an intermediate gravure cylinder or sleeve. The resonator patterns are engraved in form of a screening of $100 \mathrm{~L} / \mathrm{cm}$ (Figure 3), i.e. the resonator area consists of cavities and paths. During the transfer process of the pattern from the gravure cylinder to the substrate, a closed layer is formed. Before transferring, the rotating gravure cylinder has to be fully covered with ink (nano-particle silver ink), a doctor blade removes the excessive amount and the ink remains in the cavities only. The ink is transferred from the cavities onto a substrate (PET) which passes 
as a web between gravure and a pressure cylinder. To functionalise the nano-particle silver ink, the substrate passes an in-line post-treatment unit (in this case an infrared (IR) module) to evaporate the solvent, to cast out the organic components around the nano-particles and to stimulate a grain growth to form density and conductivity. The most important challenge, which has to be taken into account for this fabrication method, is a sufficient screening to realise the expected pattern and homogeneous layers. Additionally, the viscosity of the ink and continuously filled cavities during printing are crucial. Influential factors like pressure of the doctor blade and the pressure cylinder as well as the web speed have to be considered. Furthermore, the IR intensity has to be adjusted to the web speed to implement a homogenous drying and sintering of the printed layers with sufficient conductivity.

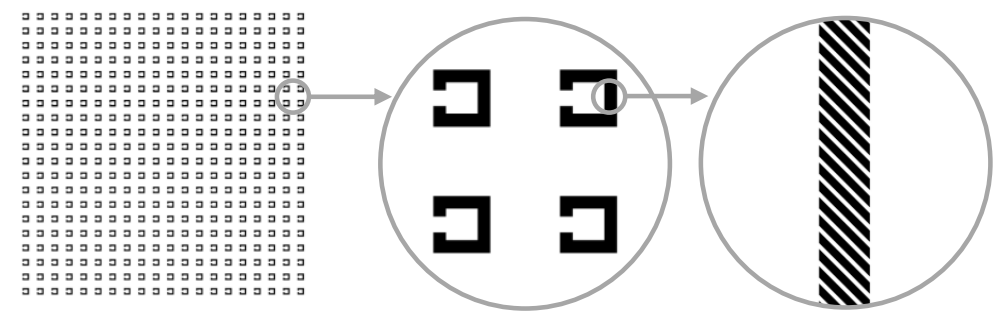

Figure 3: Demonstration of the digital pattern and screening

\subsubsection{Vacuum Infusion Technology}

The printed resonator arrays were integrated into GFRP samples with thickness values of $1.3 \mathrm{~mm}$ using the vacuum infusion technology. They are placed between stacked layers of plain woven glass fibre fabrics on a solid mould cavity and covered with a polymeric film. The resulting laminate consists of seven layers with an expected thickness of $1 \mathrm{~mm}$. Each layer has a fibre orientation of $0^{\circ}$ and $90^{\circ}$ and a grammage of $163 \mathrm{~g} / \mathrm{m}^{2}$. Two resonator arrays are placed between the first and the second layer and between the penultimate and the last layer as shown in Figure 4. To consolidate the fibre-reinforced structure, the layer stack is evacuated and infused with a thermoset plastic (epoxy resin $L$ with hardener GL 2 supplied by R\&G Faserverbundwerkstoffe $\mathrm{GmbH}$ ). The volume flow is performed by the pressure difference between vacuum and atmospheric pressure.

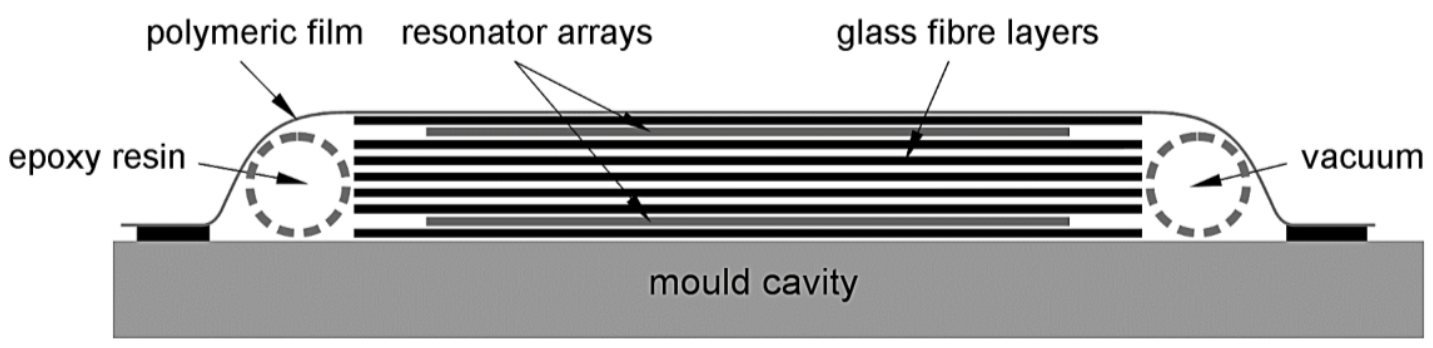

Figure 4: Schematic view of the fabrication process to create the investigated GFRP samples

\section{Results and Discussion}

Figure 5 shows the fabricated FGFRP sample with a total thickness of $1.3 \mathrm{~mm}$ and with the two integrated SSR resonator arrays. The sample is $12 \mathrm{~mm}$ long and $10 \mathrm{~mm}$ wide. 


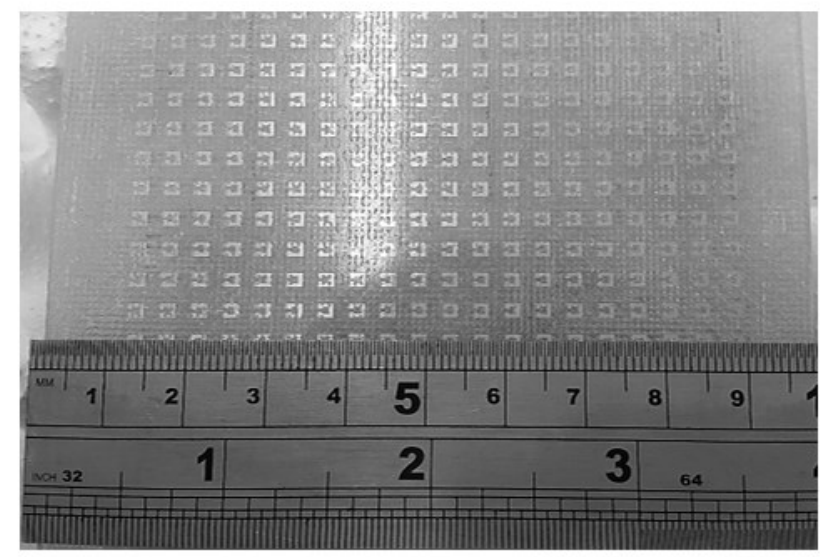

Figure 5: Fabricated FGFRP within the integrated SSR resonator arrays using gravure printing and vacuum infusion technology

A tensile test with a material test machine (type TIRATEST 28100, Tira $\mathrm{GmbH}$ ) was carried out to evaluate the behaviour of the FGFRP sample. For this purpose, the FGFRP sample was clamping into the sample holder of the material test machine. The test was driven by setting up the applied traction force on the sample. A traction force in the range from $0 \mathrm{kN}$ to $18 \mathrm{kN}$ with an increment of $1 \mathrm{kN}$ was applied to elongate the sample. The reflection response was measured using two horn antennas (type Standard Gain Horn Antenna for $24 \mathrm{GHz}$, A-Info) and a vector network analyser (type ZVA 50, Rohde \& Schwarz) after each incremental step of increasing the force. Figure $6 a$ shows the measured reflection response applying a traction force in the range from $0 \mathrm{kN}$ to $15 \mathrm{kN}$. A weaker one close to $16 \mathrm{GHz}$ and stronger one close to $21 \mathrm{GHz}$. Both reflection dips enclose a reflection peak in the range from $16.9 \mathrm{GHz}$ to $18.5 \mathrm{GHz}$.

The reflection response shows an anti-resonance at about $21 \mathrm{GHz}$ that moves to lower frequencies when the strain of the FGFRP is $2.4 \%$ (Figure $6 \mathrm{~b}$ ). The test ended with the detachment of the upper laminate layer from the integrated resonator array applying a strain of $5.5 \%$. Figure $6 \mathrm{~b}$ shows the frequency shift as a function of the applied strain driven by the applied traction force and because of the geometry of the FGFRP sample. From Figure $6 \mathrm{~b}$ it can be seen that the frequency shift starts when a strain of $2 \%$ is achieved.

The applied measurements show that the application of a printed electromagnetic resonator array integrated into a GFRP material are qualified to modify the reflection response of a normal GFRP structure. The use of two resonator arrays that are integrated into a GFRP structure at a certain distance from each other, lead to the formation of a stopband that is characterised by one broadband reflection peak enclosed by to reflection dips. The reflection peak is realised by the first order resonance mode of the resonator arrays and can mainly be modified by varying the dimension of the SSR resonators. Changing the resonance frequency of the resonators effects the location of the stopband. However, the arrangement of the resonator arrays, the material and the material thickness have an influence as well and must be considered during the design process. 


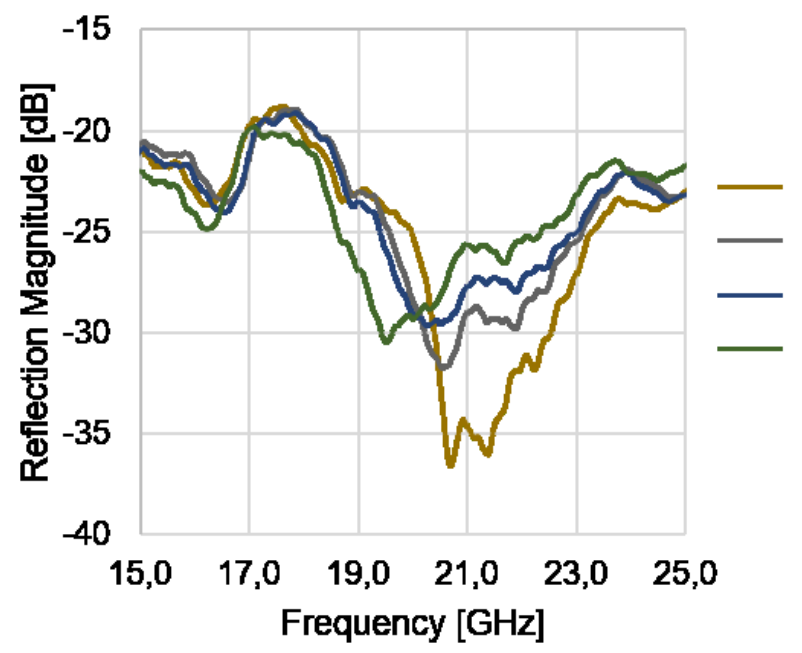

a)

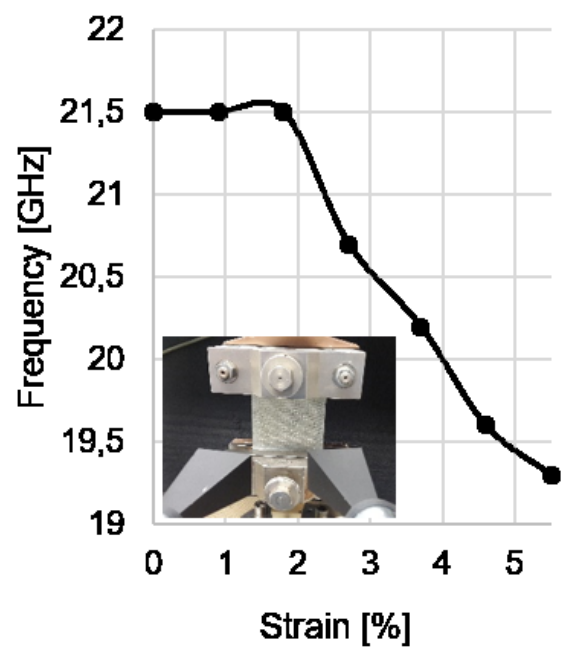

b)

Figure 6: Result of the tensile test applied on the fabricated $1.3 \mathrm{~mm}$ FGFRP sample showing a) performed reflection measurement by means of three selected reflection responses and $b$ ) the visualisation of the frequency shift depending on the applied strain and with the inset image of the used measurement instrumentation

\section{$4 \quad$ Integrated Life Cycle Engineering and Business Modelling}

In the previous sections, the potential of functionalising lightweight structures by integrating printed electromagnetic resonator arrays was shown. To fully deploy the potential of this innovative technology, the technical research and engineering activities should be integrated with business modelling (BM). This helps to direct the engineering activities towards market success and profitability of the technology. For an integrated life cycle-related engineering (including the effects on the life cycle phases of components, products etc.) and BM, the concept shown in Figure 7 can be applied [4, 11]. This concept is characterised by an intensive exchange of information for coupling the various technical and economic (additionally maybe also ecologically-oriented) design and evaluation activities: Here, the new technology is the starting point for generating business model ideas. Furthermore, the engineering results are a decisive input for the elaboration of business model elements, the strategic and the financial evaluation. The BM results in turn will influence the need and direction of engineering activities.

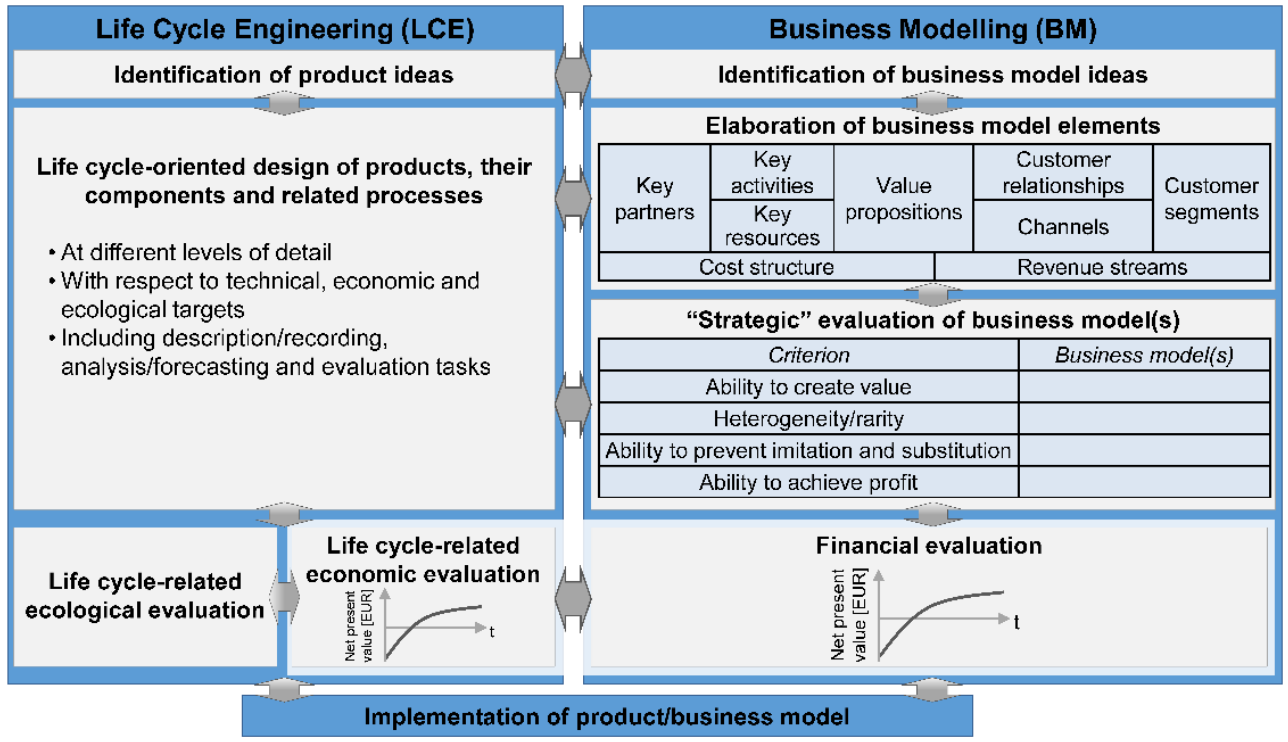

Figure 7: Concept of integrated LCE and BM ([4] developed based on [12, 13]) 
In the following, due to the early stage of technical considerations only the first step of BM is addressed (for the other steps see $[4,11]$ ): The prior business model idea refers to lightweight structures with sensitive functionality for monitoring composite materials based on GFRP. However, for elaborating, evaluating and selecting a business model it is necessary to identify, analyse, evaluate and select promising fields of application for the technology (supposing different fields exist) taking into account the technological potential (compared to competing technologies), the specific application requirements and the market characteristics (market size and growth, profit potential, competitors etc.) [14]. Therefore, the approach of an Integrated Technology, User and Market Analysis and Forecast [15] seems to be useful.

Technology Analysis and Forecast comprise the description and classification of the technology as well as the view on competing technologies. With User Analysis and Forecast, potential users in different stages of the value chain along with their requirements, demands and willingness to pay can be identified. Therefore, relevant user-specific technology-dependent costs have to be analysed by using life cycle costing and/or instruments for the cost appraisal of processes and value chains. A commercialisation can only succeed if the new technology fits the requirements and demands. Particular attention should be paid to this factor. By Market Analysis and Forecast, the market and competitors are characterised and their performance forecast. Finally, Technology Appraisal integrates the results of analyses and forecasts to an overall appraisal of the economic potential of a new technology [15].

Applying this method, condition monitoring for wind rotor blades and SHM for car body structures are identified as first promising fields of application. For each of these fields, the mentioned prior business model idea can be differentiated concerning the different components and processes necessary to provide these structures. For a systematic derivation of the corresponding "sub-ideas", two (identification and) breakdown processes are recommended: i) breaking down lightweight structures with sensitive functionality for monitoring composite materials based on GFRP into their components (product breakdown structure) and ii) identifying the processes and sub-processes required along the life cycle of the lightweight structures (life cycle-related process breakdown structure). The next steps will be to derive, elaborate and evaluate these business model ideas (for first considerations regarding the field of ice detection of wind rotor blades see [11]).

\section{Conclusion and Outlook}

The performed work demonstrates that the integration of printed electromagnetic resonator arrays allows for the functionalisation of lightweight structures and hence the realisation of smart lightweight structures with sensory function for remote and contactless structural health monitoring. The designed electromagnetic behaviour with its sensitivity against structural variation is used as a sensor function rendering the detection of material stress possible and hence performing structural health monitoring.

The performed experiments with a tensile test machine and the fabricated FGFRP sample show the potential to change the electromagnetic response of the material under stress. However, it was noticed that the sensitivity is still too weak and requires improvements. Therefore, future research activities will focus on increasing the sensitivity of the passive sensor approach using electromagnetic resonator arrays. The outlined concept of integrated business modelling will be used for elaborating and evaluating the identified business model ideas of condition monitoring for wind rotor blades and structural health monitoring for car body structures.

\section{Acknowledgement}

The work was performed within the Federal Cluster of Excellence EXC 1075 "MERGE Technologies for Multifunctional Lightweight Structures" and supported by the German Research Foundation (DFG). Financial support is gratefully acknowledged. 


\section{References}

[1] Schüller, M.; Lipowski, M; Großmann, T.; Tröltzsch, J.; Geßner, T.; Kroll, L.: Integration von Mikro- und Nanosystemen in Hybride Strukturen, Smarte Strukturen und Systeme. In: Tagungsband 4SMARTS-Symposium, 2016, pp. 161-170.

[2] Walther, M.; Kroll, L.; Stockmann, M.; Elsner, H.; Heinrich, M.; Wagner, S.: Investigation in development of embroidered strain measurement sensors. In: 10th IMEKO TC15 Youth on Experimental Solid Mechanics, 2011, pp. 117-118.

[3] Hartwig, M.; Gaitzsch, M.; Großmann, T.D.; Heinrich, M.; Kroll, L.; Gessner, T.; Baumann, R. R.: Investigation on Inkjet Printed Passive Sensors for Wireless Ice Detection on Wind Rotor Blades. J. of Imaging Sci. and Technol., 60(4) (2016), pp. 40402-1 - 404002-7. doi: 10.2352/J.ImagingSci.Technol.2016.60.4.040402

[4] Götze, U.; Peças, P.; Schmidt, A.; Symmank, C.; Henriques, E.; Ribeiro, I.; Schüller, M.: Life Cycle Engineering and Management - Fostering the Management-orientation of Life Cycle Engineering Activities. Procedia CIRP, 61 (2017), pp.134-139. doi: 10.1016/j.procir.2016.11.240

[5] Schubert, L.; Klesse, T.; Weihnacht, B.; Schulze, E.; Lieske, U.; Frankenstein, B.: Aktuelle Entwicklungen zu aktiv angeregten geführten Wellen (Acousto-Ultransonics) für SHMAnwendungen. In: 20. Kolloquium Schallemission, 18. - 19. Juni 2015, Garmisch-Partenkirchen, 2015.

[6] Schubert, L.: Zustandsüberwachung an Faserverbundwerkstoffen mit geführten Wellen. ZfPZeitung 136, Oktober 2013, pp. 52-58.

[7] Jang, S.; Kim, J.: Passive wireless structural health monitoring sensor made with a flexible planar dipole antenna. Smart Mater. Struct., 21 (2012), pp. 1-6. doi: 10.1088/0964-1726/21/2/027001

[8] Wilson, W.; Rogge, M.; Fisher B.;Malocha, D.; Atkinson, G.: Fastener Failure Detection Using a Surface Acoustic Wave Strain Sensor. IEEE Sens. J., 12(6) (2012), pp. 1993-2000. doi: 10.1109/JSEN.2011.2181160

[9] Zhongqing, B.; Hara, M.; Mitsui, M.; Sano, K.; Nagasawa, Sumito, Kuwano, H.: Experimental Study of High Sensitive Sensor Using a Surface Acoustic Wave Resonator for Wireless Strain Detection. Jpn. J. of Appl. Phys., 51 (2012), 2012. pp. 07GC23-1 - 07GC23-4. doi: 10.1143/JJAP.51.07GC23

[10] Melik, R.; Unal, E.; Perkgoz, N. K.; Puttlitz, C.; Demir, H. V.: Metamaterial-based wireless strain sensors. Appl. Phys. Lett., 95 (2009). pp. 011106-1 - 011106-3 doi: 10.1063/1.3162336

[11] Götze, U.; Symmank, C.; Schmidt, A.; Großmann, T. D.; Schüller, M.; Kurth, S.; Otto, T.: Integrierte Geschäftsmodell- und Technologieentwicklung für smarte Systeme und Strukturen. In: Wiedemann, M.; Melz, T. (eds.): Smarte Strukturen und Systeme. Tagungsband des 4SMARTSymposiums, Braunschweig, 21.-22. Juni 2017, Aachen: Shaker, 2017, pp. 401-413.

[12] Rehme, M.; Lindner, R.; Götze, U.: Perspektiven für Geschäftsmodelle der Fahrstrombereitstellung - Wirtschaftliche Bewertung in elektromobilen Supply Chains. In: Proff, $\mathrm{H}$. (ed.): Entscheidungen beim Übergang in die Elektromobilität - Technische und betriebswirtschaftliche Aspekte, Wiesbaden: Springer Gabler, 2015, pp. 409-428.

[13] Osterwalder, A.; Pigneur, Y.: Business Model Generation: A Handbook for Visionaries, Game Changers, and Challengers, Hoboken, New Jersey: John Wiley \& Sons, 2010.

[14] Götze, U.; Schmidt, A.; Herold, F.; Nestler, D.; Siebeck, S.: Methodik zur Analyse, Prognose und Bewertung von innovativen Werkstoffen am Beispiel von partikelverstärkten AluminiummatrixVerbundwerkstoffen (AMCs). In: Gausemeier, J. (ed.): Vorausschau und Technologieplanung. Heinz Nixdorf Institut, Paderborn, 2015, pp. 221-241.

[15] Götze, U.; Schmidt, A.: Innovation Control - Framework, Methods, and Applications. In: Nistor, R.; Zaharie, M.; Gavrea, C. (eds.): Managerial Challenges of the Contemporary Society, vol. 5, Cluj-Napoca, Romania, 2013, pp. 100-105. 